\title{
Uveitis Associated with Primary Angiitis of the Central Nervous System
}

\author{
A.R. Woolfenden, N.K. Wade, P. Tang, A. Chalmers, G. Reid, P.A. Teal
}

\begin{abstract}
Background: In Primary Angiitis of the Central Nervous System (PACNS), disease is typically limited to the brain and spinal cord although other organs may be affected. Uveitis is occasionally seen in systemic vasculitides but is not a recognized manifestation of PACNS. We describe two patients who developed PACNS following the onset of uveitis. Case Descriptions: Case 1A 47-year-old male suffered multiple TIAs and left pontine stroke shortly after two episodes of diffuse uveitis. A cerbral angiogram demonstrated multiple caliber changes within several intracranial vessels. Cyclophosphamide was added after his stroke occurred during pulse methylprednisolone therapy. Case 2- A 35-year-old male suffered a spinal cord TIA followed by hemispheric and brainstem infarctions two months after an episode of uveitis and Bell's palsy treated with oral prednisone. A cerebral angiogram demonstrated multiple caliber changes within several intracranial vessels. He was successfully treated with oral prednisone and cyclophosphamide. Conclusions: Uveitis should be considered a recognized feature of PACNS. Combination immunosuppressive therapy with prednisone and cyclophosphamide may be necessary for successful treatment.
\end{abstract}

RÉSUMÉ: Uvéite associée à une angéite primaire du système nerveux central. Contexte : Dans l'angéite primaire du système nerveux central (APSNC), il est typique d'observer une atteinte limitée au cerveau et à la moelle épinière, bien que d'autres organes puissent être touchés. On peut occasionnellement observer une uvéite dans les vasculites systémiques, mais l'uvéite n'est pas une manifestation reconnue de l'APSNC. Nous décrivons les cas de deux patients qui ont développé une APSNC suite à une uvéite. Observations : Observation 1 : Un homme de 47 ans a présenté de multiples épisodes d'accidents ischémiques transitoires (AIT) et un accident vasculaire cérébral peu après deux épisodes d'uvéite diffuse. Un angiogramme cérébral a mis en évidence de multiples changements de calibre au niveau de plusieurs vaisseaux intracrâniens. On a ajouté du cyclophosphamide à sa corticothérapie (méthylprednisolone) à doses en perfusions intraveineuses rapides. Cas 2 : Un homme de 35 ans a présenté une AIT à la moelle épinière suivie d'infarcissement hémisphérique et du tronc cérébral deux mois après un épisode d'uvéite et de paralysie de Bell traité par la prednisone par voie orale. À l'angiogramme, on a observé de multiples changements de calibre de plusieurs vaisseaux intracrâniens. Il a été traité avec succès par la prednisone et la cyclophosphamide par voie orale. Conclusions : L'uvéite devrait être reconnue comme une manifestation possible de l'APSNC. Il peut être nécessaire d'avoir recours à un traitement immunosuppresseur combinant la prednisone et la cyclophosphamide pour assurer le succès du traitement.

Can. J. Neurol. Sci. 2007; 34: 81-83

Primary Angiitis of the Central Nervous System (PACNS) is an idiopathic, typically granulomatous, inflammatory vasculopathy which causes a wide spectrum of neurologic symptoms and signs. ${ }^{1}$ Primary Angiitis of the Central Nervous System is generally confined to the central nervous system, although some patients have elevated erythrocyte sedimentation rates (ESR), and autopsy data has demonstrated disease outside the CNS. ${ }^{1-3}$ Optic neuritis and retinal vasculitis are rare but reported associations. ${ }^{1,4}$

We describe two patients with uveitis in whom PACNS subsequently developed, manifested by headache and ischemic stroke. Both required aggressive immunosuppression for successful treatment.

\section{CASe Reports}

\section{Case 1:}

A 35-year-old male developed left arm weakness and sensory loss without other associated symptoms. Two months previously he experienced a febrile illness with generalized headache. One week thereafter he developed right lower motor neuron facial weakness and bilateral, left greater than right anterior uveitis diagnosed by an ophthalmologist. A head computed tomography (CT) scan and lumbar puncture were normal. He was briefly treated with oral and topical prednisone during which time the headaches improved. One week after initiating prednisone, he suffered a transient episode of bilateral lower extremity weakness that resolved over several hours. Two weeks prior to the onset of the left hemiparesis, he had a brief episode of

From the Division of Neurology (ARW, PAT), Ophthalmology (NKW), Rheumatology (PT, AC, GR), Department of Medicine, University of British Columbia, Vancouver, BC, Canada.

ReCEIVEd OCtOBER 30, 2001. ACCEPTED IN FINAL FORM OCTOBER 10, 2006. Reprint requests to: Andrew R. Woolfenden, S\#208-888 West 8th Avenue, Vancouver, British Columbia, V5Z 3Y1, Canada. 
nausea, lightheadedness and diplopia. At the time of the most recent symptoms, the generalized headache had worsened. There was no significant past medical history.

On neurological examination, he had right lower motor neuron facial weakness, no left facial weakness, and a moderate left hemiparesis. Left-sided reflexes were brisk with an associated "upgoing" plantar response. On slit lamp, the uveitis had resolved. The general examination was normal.

A CT head scan demonstrated a small infarction in the right motor strip. Magnetic resonance imaging (MRI) confirmed the right cortical infarction and also demonstrated ischemic lesions in the left midbrain and right thalamus. A cerebral angiogram demonstrated irregularities in several intracranial vessels. CBC, ESR, renal and liver function tests, and complement levels were normal. ANA and ENA were negative. In the CSF, there was a mild pleocytosis (wbc $28 \times 10^{6} / \mathrm{L} ; 4 \%$ polymorphs, $81 \%$ lymphocytes, $15 \%$ monocytes) with a normal protein and glucose.

The patient was started on oral prednisone $1 \mathrm{mg} / \mathrm{kg} / \mathrm{d}$ and cyclophosphamide $150 \mathrm{mg}$ daily for presumed vasculitis. Over the course of the next six months, the prednisone was gradually tapered and the cyclophosphamide was continued. The headache and lower facial palsy quickly resolved, and the hemiparesis significantly improved. No new lesions were identified on follow-up MRI and a cerebral angiogram returned to normal at seven months (Figure). The immunosuppressive medications were stopped and the patient remained well at follow-up four months later.

\section{Case 2:}

A 47-year-old male was transferred to our hospital for the evaluation of multiple transient ischemic attacks. At age 35, he developed migraine with visual aura. He suffered one migraine headache every two years but in the preceding three months, he experienced three migraine headaches per week.

Apart from migraine, he had been healthy until three months prior when he suffered a febrile illness, $8 \mathrm{~kg}$ weight loss and eye redness with pain, diagnosed as bilateral nongranulomatous diffuse uveitis with multifocal choroiditis by a uveitis specialist. He was treated with topical prednisolone acetate $1 \%$, cyclopentolate $1 \%$, and diclofenac $0.03 \%$ which led to resolution of his uveitis. However, two weeks prior to the current presentation, the uveitis recurred and was again controlled with topical medications.
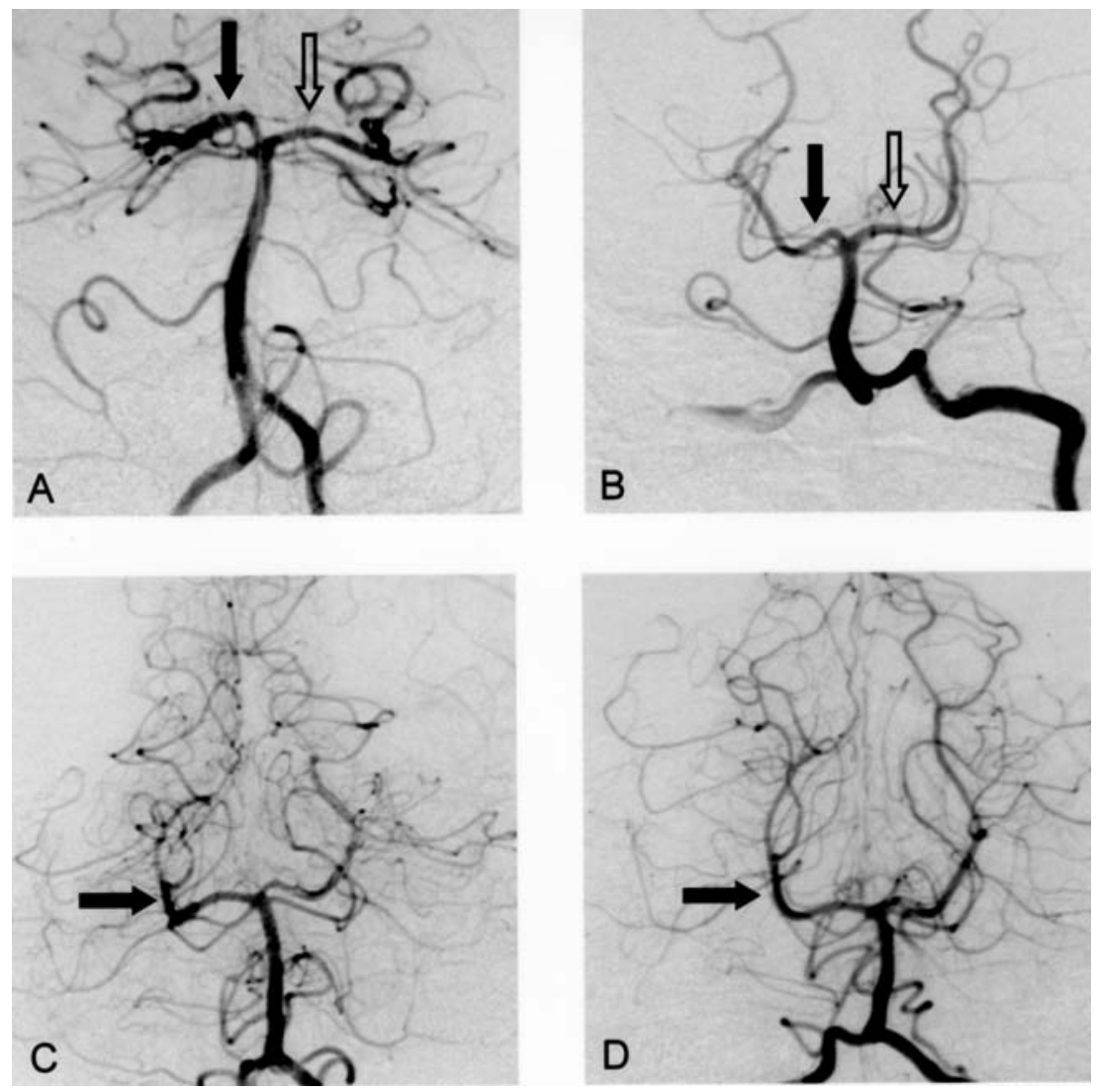

Figure: Initial $(A)$ and follow-up $(B)$ cerebral angiogram of Case 1 showing resolution of the bilateral proximal posterior cerebral artery caliber changes following immunosuppressive therapy. Initial $(C)$ and follow-up $(D)$ cerebral angiography of Case 2 showing significant improvement in the subtle right posterior cerebral artery caliber changes following immunosuppressive therapy. 
On the day of presentation, he had a typical migrainous visual aura while driving. Several minutes later, he developed sudden right-sided numbness and weakness which lasted several minutes, resolved, and then recurred a few minutes later. His speech was slurred but comprehensible. There was no headache. He improved over 5-10 minutes and went to a local hospital. There the symptoms returned briefly for a third time and he was transferred to our hospital. His general and neurologic examination was normal. The patient was started on intravenous heparin and oral aspirin after a normal head CT scan.

Routine bloodwork was normal. The ESR was $18 \mathrm{~mm} /$ hour. Serum rheumatologic markers including rheumatoid factor (RF), antinuclear antibody titre (ANA), HLA-B27, extractable nuclear antigens (ENA), cryoglobulins, antineutrophil cytoplasmic antibody (ANCA), and complement levels were unremarkable. A chest radiograph and angiotensin converting enzyme level were normal. Serology for HIV, Lyme, toxoplasmosis, hepatitis, and syphilis were negative. There was a pleocytosis in the CSF (wbc $36 \times 10^{6} / \mathrm{L} ; 6 \%$ polymorphs, $81 \%$ lymphocytes, $13 \%$ monocytes) with normal protein, glucose, and cultures. CSF VDRL and oligoclonal banding were negative.

The patient suffered several further episodes of brief hemiparesis over the next few hours prompting an urgent cerebral angiogram. This demonstrated diffuse multiple distal vessel caliber changes consistent with vasculitis. The proximal cervical arteries were normal.

The heparin was stopped, aspirin $325 \mathrm{mg} / \mathrm{d}$ was continued, and the patient was started on intravenous pulse methylprednisolone $1 \mathrm{~g}$ daily. Following the second dose, the patient developed a persistent right hemiparesis. The MRI demonstrated infarctions in the left basis pons, right frontoparietal, and left parieto-occipital regions. He was started on oral prednisone $80 \mathrm{mg}$ daily and intravenous pulse cyclophosphamide monthly. The aspirin was continued.

Over the next six months, the prednisone was tapered and the cyclophosphamide continued. The headaches and hemiparesis resolved, no new neurologic symptoms occurred, and there were no further episodes of uveitis. At six months, a repeat MRI revealed one new lesion in the left middle cerebellar peduncle. A repeat angiogram demonstrated slight improvement in the posterior cerebral artery changes (Figure). A mild pleocytosis (wbc 7 x 106/L; 94\% lymphocytes, 6\% monocytes) persisted. On this basis, the pulse cyclophosphamide was continued for a further three months with normalization of the CSF. There were no recurrent symptoms 12 months following cessation of immunosuppression and only subtle right-sided motor findings could be found on neurologic examination.

\section{Discussion}

We are aware of only one other report describing an association between PACNS and uveitis. ${ }^{5}$ Rosenbaum et al described three patients with a similar idiopathic syndrome, including one patient with biopsy confirmed CNS granulomatous angiitis. In two of three patients, neurologic symptoms occurred at the same time as the uveitis. Two patients deteriorated on steroids and improved after cyclophosphamide was started, similar to our patients. All were treated with prednisone and cyclophosphamide. Two patients had a complete recovery and one patient suffered progressive dementia and eventually died.
Diagnosis and treatment of isolated PACNS is somewhat contentious. ${ }^{6,7}$ Many consider the combination of prednisone and cyclophosphamide necessary although there may exist a more benign syndrome termed benign angiopathy of the CNS (BACNS) in which cyclophosphamide is not required ${ }^{8}$ However some patients whose clinical presentation resembles BACNS, do not follow a benign course and require more aggressive immunosuppression. ${ }^{9}$ In patients with the PACNS/uveitis syndrome reported thus far, four of five patients initially treated with prednisone alone suffered strokes. Therefore, it appears that the combination of prednisone and cyclophosphamide are required to suppress disease activity in the PACNS/uveitis syndrome.

There are several syndromes that may mimic uveitis/PACNS including infection (HIV, HTLV-1 Syphilis, Lyme, Herpes simplex, Cytomegalovirus, Toxoplasmosis), malignancy (lymphoma, including intravascular lymphoma), medical diseases (Sarcoidosis, Behcet's, Vogt-Koyanagi-Harada syndrome), and systemic vasculitis. ${ }^{5}$ Most can be ruled out clinically or by appropriate investigations. Neither of our patients appeared to have a secondary cause for their syndrome although we could not fully exclude a possible association with an unidentified infectious agent.

We speculate that an immune mediated mechanism may explain why uveitis is associated with PACNS. In all cases, the uveitis is bilateral. In addition, uveitis is sometimes observed in experimental allergic encephalitis models. ${ }^{10}$ The concomitant occurrence of uveitis and PACNS may be the result of shared vascular endothelium, neural antigens, pial lining, and immune privilege between the eye and brain.

In summary, uveitis should be recognized as a feature of PACNS. Primary Angiitis of the Central Nervous System should be considered in patients who present with uveitis and stroke. After excluding secondary causes, such patients appear to require combination immunosuppressive therapy with prednisone and cyclophosphamide for successful treatment.

\section{REFERENCES}

1. Hankey GJ. Isolated angiitis/angiopathy of the central nervous system. Cerebrovasc Dis. 1991;1:2-15.

2. Abu-Shakra M, Khraishi M, Grosman H, Lewtas J, Cividino A, Keystone EC. Primary angiitis of the CNS diagnosed by angiography. Q J Med. 1994;87:351-8.

3. Lie JT. Primary (Granulomatous) Angiitis of the central nervous system: A clinicopathologic analysis of 15 new cases and a review of the literature. Hum Pathol. 1992;23:164-71.

4. Vine AK. Retinal vasculitis. Sem Neurol. 1994;14:354-60.

5. Rosenbaum JT, Roman-Goldstein S, Lindquist GR, Rosenbaum RB. Uveitis and central nervous system vasculitis. J Rheumatol. 1998;25:593-7.

6. Vollmer TL, Guarnaccia J, Harrington W, Pacia SV, Petroff OAC. Idiopathic granulomatous angiitis of the central nervous system: Diagnostic Challenges. Arch Neurol. 1993;50:925-30.

7. Moore PM. Diagnosis and management of isolated angiitis of the central nervous system. Neurology. 1989;39:167-73.

8. Calabrese LH, Gragg LA, Furlan AJ. Benign angiopathy: A distinct subset of angiographically defined primary angiitis of the central nervous system. J Rheumatol. 1993;20:2046-50.

9. Woolfenden AR, Tong DC, Marks MP, Ali AO, Albers GW. Angiographically defined primary angiitis of the central nervous system: Is it really benign? Neurology. 1998;51:183-8.

10. Shikishima K, Lee WR, Behan WM, Foulds WS. Uveitis and retinal vasculitis in acute experimental allergic encephalomyelitis in the Lewis rat: an ultrastructural study. Exp Eye Res. 1993;56: 167-75. 